\title{
NUMERICAL SOLUTION OF POLLUTION OF GROUNDWATER
}

\author{
RADKO PETKOV* \\ Institute of Mechanics, Bulgarian Academy of Sciences, \\ Acad. G. Bonchev Str., Block 4, Sofia 1113, Bulgaria
}

[Received: 11 March 2019. Accepted: 13 May 2019]

doi: 10.7546/JTAM.49.19.02.06

\begin{abstract}
The present work describes the obtained mathematical model of non-steady filtration thermal dispersion, taking into account the changes in the fluid density and viscosity depending on temperature and concentration of the contained admixtures.

A method has been developed for evaluating the dispersion coefficients in porous media. An original hybrid numerical method has been developed on the basis of the finite element and finite difference methods for pressure-head and non-pressure filtration for solving the problems of filtration thermal dispersion in porous media.

The "Nonsteady_Termo_Dispersion-RP" software, operated in Windows environment and visualizing the results using MATLAB has been developed. The software has been applied for real conditions with the respective input data. As a result of the numerical experiments, data have been obtained for the effect of density and viscosity of sodium chloride, ammonium nitrate depending on the equations of state in filtration dispersion in porous media. The degree of substantiation and reliability of the scientific achievements is ensured by the use of a complemented hydrodynamic model, based on the fundamental equations of filtration, dispersion and thermal conductivity. The reliability of the results is also ensured by the fact that absolutely stable and convergent numerical schemes are used in the realized numerical experiments [1].
\end{abstract}

KEY WORDS: numerical modelling, groundwater pollution, filtration thermodispersion, porous media

\section{INTRODUCTION}

In the recent years protection of environment and its recovery are considered to be one of the most important problems, the solving of which stimulates scientific progress. It is known that solid and liquid wastes from industrial production represent basic pollution sources of water resources (rivers, seas, lakes and groundwater) [2,3]. With

${ }^{*}$ Corresponding author e-mail: radolili@yahoo.com 
respect to chemical composition the harmful substances are rather diverse - a result of the different existing industrial technologies. In mining industry, where slags containing solid and liquid contaminants are released, tailing ponds are usually built with hydraulic discharge of wastes [4]. The wastes released from the factories of non-ferrous metallurgy contain considerable amounts of chemical substances too. In the big plants the tailing (slag) ponds occupy large sites and it is known from practice that they also pollute the environment. It is just in the regions, where similar wastes are disposed, that the majority of the cases with groundwater pollution are observed. During filtration the industrial wastewater contaminates the upper aquifer horizons in these regions. If there is a hydraulic connection between the layers, the polluted aquifer horizons from the surface may reach deeper layers. For this reason, during the design of the locations for harmful substance deposition, it is necessary to make preliminary investigation of the eventual contamination and to envisage measures for its prevention.

One of the major hydrogeological problems is to protect fresh groundwater as one of the sources of drinking water supply from pollution ensuing from soil fertilizers. The main hydrogeological problems are to study the conditions of groundwater contamination, the degree of water protection and the changes in water quality, to establish the area and scale of pollution, to investigate the regularities of contaminant transport in groundwater, as well as to predict the process of pollution.

2 Hydrodynamic Model of Thermodispersion of NON-CONSERVATIVE AdMIXTURES IN PoROUS MEDIA

1. Differential equation, describing a 3-non-stationary convective-dispersive transfer in a dimensionless form

$$
\text { (1) } \begin{aligned}
\frac{\partial}{\partial x}\left(D_{x x} \frac{\partial}{\partial x}\left(\frac{C}{\rho}\right)\right)+ & \frac{\partial}{\partial y}\left(D_{y y} \frac{\partial}{\partial y}\left(\frac{C}{\rho}\right)\right)+\frac{\partial}{\partial z}\left(D_{z z} \frac{\partial}{\partial z}\left(\frac{C}{\rho}\right)\right) \\
& -\frac{\partial}{\partial x}\left(u_{x} C\right)-\frac{\partial}{\partial y}\left(u_{y} C\right)-\frac{\partial}{\partial z}\left(u_{z} C\right)-\psi=\frac{\partial C}{\partial t} .
\end{aligned}
$$

2. Differential equation, describing a 3-D non-stationary filtration in a dimensionless form

$$
\frac{\partial}{\partial x}\left(M_{x} \frac{\rho}{v} \frac{\partial H}{\partial x}\right)+\frac{\partial}{\partial y}\left(M_{y} \frac{\rho}{v} \frac{\partial H}{\partial y}\right)+\frac{\partial}{\partial z}\left(M_{z} \frac{\rho}{v} \frac{\partial H}{\partial z}\right)=S \frac{\partial H}{\partial t} .
$$

3. Equation of heat conductivity

$$
\sigma \frac{\partial T}{\partial t}+\frac{\partial}{\partial x}\left(u_{x} T\right)+\frac{\partial}{\partial y}\left(u_{y} T\right)+\frac{\partial}{\partial z}\left(u_{z} T\right)=\alpha\left(\frac{\partial^{2} T}{\partial x^{2}}+\frac{\partial^{2} T}{\partial y^{2}}+\frac{\partial^{2} T}{\partial z^{2}}\right) .
$$




\section{Equation of state}

$$
\rho=\rho(C, T), \quad \nu=\nu(C, T),
$$

where $C(x, y, z, t)$ is the concentration of solute substances; $u_{x}, u_{y}, u_{z}-$ the dimensionless components of the velocity vector in the respective directions; $T$ - the temperature of the entering solute substances, $D_{x x}, D_{y y}, D_{z z}$ - the coefficients of hydrodynamic dispersion [5]; $H(x, y, z, t)$ is the dimensionless pressure-head of the movement; $y$ - the source of pollution; $k_{n x}, k_{n y}, k_{n z}$ - the coefficients of permeability along the directions, and $z ; L_{1}, L_{2}, L_{3}$ - the linear lengths, determining the filtration area; $t^{*}$ - the time period of investigated migration; $M_{x}, M_{y}, M_{z}$ - the dimensionless parameters; $x, y, z-$ the dimensionless coordinates; $\nu$ and $\rho$ - the kinematic viscosity and the density of the filtration flux, which depend on the concentration of migrating substances in case of active admixtures or are constants in case of migrating passive admixtures.

For convenience in the calculations, equations (1), (2), (3), (4) are transformed in a dimensionless form by introducing the following dimensionless parameters:

$$
\begin{array}{llll}
\bar{x}=\frac{x}{L_{1}}, & \bar{y}=\frac{y}{L_{2}}, & \bar{z}=\frac{z}{L_{3}}, & \bar{\rho}=\frac{\rho}{\rho_{0}}, \\
\bar{\nu}=\frac{\nu}{\nu_{0}}, & \bar{C}=\frac{C}{C_{0}}, & \bar{H}=\frac{H}{H_{0}}, & \bar{t}=\frac{t}{t^{*}}, \\
\bar{D}_{x x}=\frac{D_{x x} t^{*}}{L_{1}^{2}}, & \bar{D}_{y y}=\frac{D_{y y} t^{*}}{L_{2}^{2}}, & \bar{D}_{z z}=\frac{D_{z z} t^{*}}{L_{3}^{2}} &
\end{array}
$$

for equations (1), (2), (4) and

$$
\bar{T}=\frac{T}{T_{0}}, \quad \bar{\alpha}=\frac{t^{*} \alpha}{L_{1}^{2}}, \quad \bar{t}=\frac{t}{t^{*}}, \quad \bar{u}_{x}=\frac{u_{x} L_{1}}{t^{*}}, \quad \bar{x}=\frac{x}{L_{1}}
$$

for equation (3).

The equation of Darcy for filtration velocity is

$$
u_{x}=-\frac{k_{x} g}{\nu n} \frac{\partial H}{\partial x}, \quad u_{y}=-\frac{k_{y} g}{\nu n} \frac{\partial H}{\partial y}, \quad u_{z}=-\frac{k_{z} g}{\nu n} \frac{\partial H}{\partial z},
$$

$M_{x}=\frac{k_{n x} g t^{*} H_{0}}{\nu_{0} n_{0} L_{1}^{2}}, M_{y}=\frac{k_{n y} g t^{*} H_{0}}{\nu_{0} n_{0} L_{2}^{2}}, M_{z}=\frac{k_{n z} g t^{*} H_{0}}{\nu_{0} n_{0} L_{3}^{2}}$ in (2) are dimensionless quantities.

The coefficient of thermal conductivity $\alpha=\frac{\xi}{\rho C_{\rho}}$ and the coefficient $\sigma$ has the form

$$
\sigma=\frac{\left[\left(\rho C_{\rho}\right)_{f}+\left(\rho C_{\rho}\right)_{m}\right]}{\left(\rho C_{\rho}\right)_{f}}
$$


where $C_{\rho}$ is the released heat of the fluid at constant pressure; $\xi$ - the coefficient of thermal conductivity for the "fluid-porous medium" system, $f$ refers to the fluid and $m$ - to the porous medium. The functions $\rho=\rho(C, T)$ and $\nu=\nu(C, T)$ in (4) are determined experimentally for each particular pollutant [6].

Prior to defining the initial and boundary conditions, we have to note that in the case of non-conservative solute substance migration the equations (1), (2), (3), and (4) cannot be solved independently of each other due to the mutual interrelation between the concentration in (1) and the velocity field in (5), in contrast to the case with conservative solute substance migration, when such an approach is possible. This is important and brings certain difficulties that have to be overcome in the numerical solution of the system of equations.

\section{INITIAL AND BOUNDARY CONDITIONS}

The respective initial and boundary conditions (Fig. 1) have to be set in order to find the solution of the system of differential equations (1), (2), (3), and (4) in a given domain. They depend on the particular conditions of the problem in this domain and determine a partial solution of the problem. The definition of the initial and boundary conditions is a very important and responsible task, especially in the investigation of filtration and migration processes in complex and large regions. The aspect of the difficulties in determining the initial and boundary conditions will not be treated here

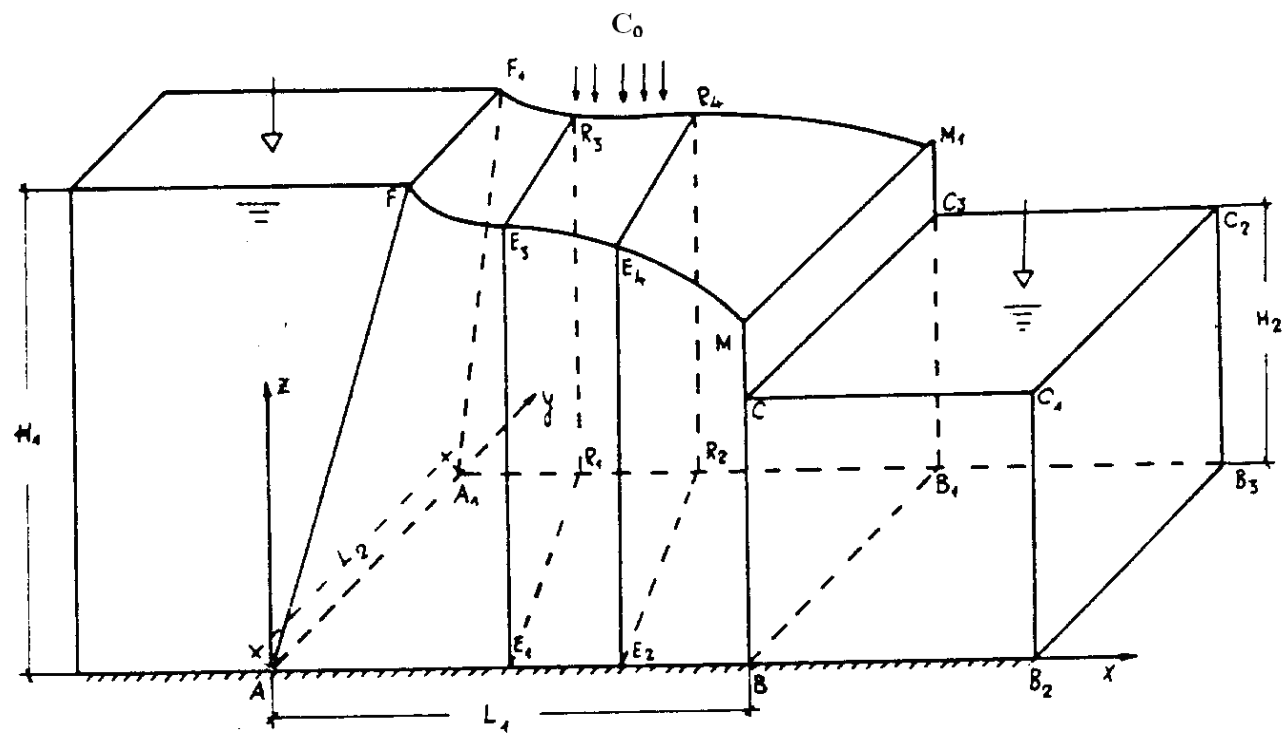

Fig. 1. The solution domain. 
since the objective is to give an optimization approach to solving the inverse problem.

We search for the solution of the problem in the variable domain $\Omega(t)$, determined by the condition

(7) $\Omega(t)=\left\{(x, y, z)\left[\varphi(x, y) \leq z \leq H_{1} ; 0 \leq x \leq L_{1} ; 0 \leq y \leq L_{2}\right]\right\} \quad 0<t<\tau$.

The initial and boundary conditions of (1) and (2) in this domain are assigned in accordance with the explanations and designations in Fig. 1

$$
\begin{array}{cl}
H(x, y, x, 0)=H_{0}(x, y, z, 0) ; & C(x, y, z, 0)=0 \text { in } \Omega(0) \\
H=z_{1}(t) \text { on } A A_{1} F F_{1} ; & H=z_{2}(t) \text { on } B C C_{3} B_{1} \\
H=z \text { on } F M F_{1} M_{1} ; & C=C_{0}(t) \text { on } R_{3} R_{4} E_{3} E_{4} \\
\frac{\partial H}{\partial t}=\frac{1}{S}\left(u_{x} \tan \alpha+u_{y} \tan \beta+u_{z}\right) \text { on } \Gamma(t) \\
\Gamma(t)=\left\{(x, y, z)\left[z=\phi(x, y, t) ; 0 \leq x \leq L_{1} ; n \leq y \leq L_{2}\right]\right\} \\
\frac{\partial H}{\partial n}=0 ; \quad \frac{\partial C}{\partial n}=0 ; \quad \frac{\partial T}{\partial n}=0
\end{array}
$$

on $A B B_{1} A_{1}, A B M F$ and $A_{1} B_{1} M_{1} F_{1}$.

$$
\begin{array}{ll}
\frac{\partial C}{\partial n}=0 ; \quad \frac{\partial T}{\partial n}=0 & \text { on } M B B_{1} M_{1} \\
\frac{\partial C}{\partial n}=0 ; \quad \frac{\partial T}{\partial n}=0 & \text { on } A A_{1} F F_{1} \\
\frac{\partial T}{\partial n}=T_{0} \frac{\partial \phi}{\partial n} & \text { on } \Gamma(t), \quad 0<t \leq \tau,
\end{array}
$$

where $\varphi(x, y, z)$ is a free boundary, which is obtained from the solution of equation (2) with the boundary and initial conditions (8), (9), (10) and (11), (12); and $\alpha$ and $\beta$ are the angles, closed between the normal $n$ to $\varphi(x, y, z)$ and the axes $x$ and $y$.

The equations (1) - (4), together with the initial and boundary conditions (8) - (16), define the hydrodynamic model of distribution of the non-conservative admixtures in a three-dimensional non-stationary pressure-head free filtration flux. A hybrid numerical model based on FEM and FDM [7, 8] is used for the numerical solution of the hydrodynamic model. The $\mathrm{C}^{++}$programming language is used for the numerical realization.

\section{InVEstigation of SALt WATER INTRUSion}

The intensive exploitation of groundwater is a major factor, determining the intrusion of marine water in aquifer horizons in the immediate proximity of seas. In its turn, 
this provokes lowering of the groundwater table and replacement of fresh by salt water [9].

Other reasons for marine water intrusion may be mining activities, mineral resource extraction, drying and oil and gas deposit exploitation. In general - all anthropogenic activities, connected with disturbances of the hydrodynamic and mechanical equilibrium of the interrelated "aquifer horizons - sea" system.

Salinization exerts impact mainly on shallow aquifer horizons with or without pressure-head, which have direct hydraulic connection with the sea or the rivers running into the sea. The intrusion of such water in the pressure-head aquifer horizons is realized via natural lithological windows or compromised boreholes, in which there is an artificial hydraulic connection with the embedded on top (otherwise well insulated) aquifer layers. In general, intrusion affects mainly groundwater and the aquifer horizons most close to the surface [10].

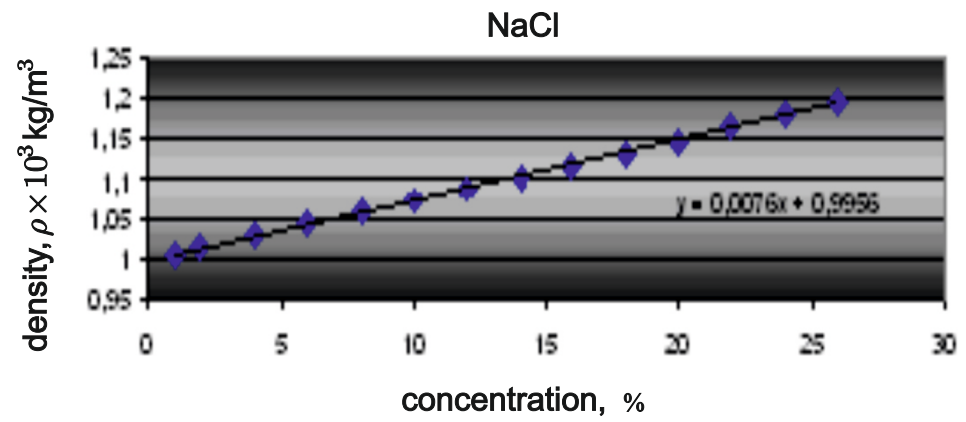

Fig. 2. Density of a sodium chloride aqueous solution depending on concentration at $20^{\circ} \mathrm{C}$.

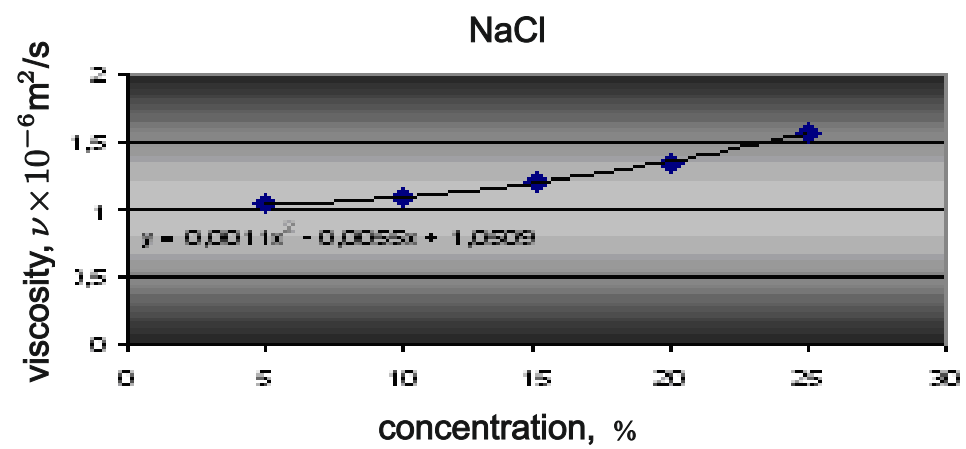

Fig. 3. Kinematic viscosity of sodium chloride aqueous solution depending on concentration at $20^{\circ} \mathrm{C}$. 
To predict salt-water intrusion, it is necessary to know the boundary, determining the differences in the density of salt and fresh water. The theoretical investigations do not reflect with sufficient reliability the real contact between marine and ground water, since the boundary is assumed to be abrupt (with a jump-like change of density). In fact the transition from the one type of water to the other is smooth, with gradual change of density [4].

The length of the marine water "tongue" depends on the gradient of the natural groundwater stream flowing into the sea, the ratio between the density of fresh and salt water and the thickness of the aquifer horizon.

The author investigates the effect of the changes in density and viscosity depending on the concentration of the infusing salt water during the process of convective dispersion in a uniform pressure-head aquifer horizon. The dimensionless parameters are used input data. The dimensions of the domain and the place of marine water infusion are arbitrarily chosen. The equations of state $f_{1}=\rho(c)$ and $f_{2}=\nu(c)$ for sodium chloride are plotted in Fig. 2 and Fig. 3.

The following input data are used for the tests in Figs. 4 and 5:

$M_{x}=0.0457886, M_{y}=0.019554, M_{z}=0.00437, H=0.3, D_{x}=0.001157$, $D_{y}=0.029554, D_{z}=0.0052311, A_{1}=0, A_{2}=0.0076, A_{3}=1, B_{1}=0.0010$, $B_{2}=-0.0052, B_{3}=1, C_{0}=1$ for $0.55 \leq y \leq 0.66$ and $C_{0}=0.4$ for $0.91 \leq y \leq$ $0.96, t^{*}=100$.

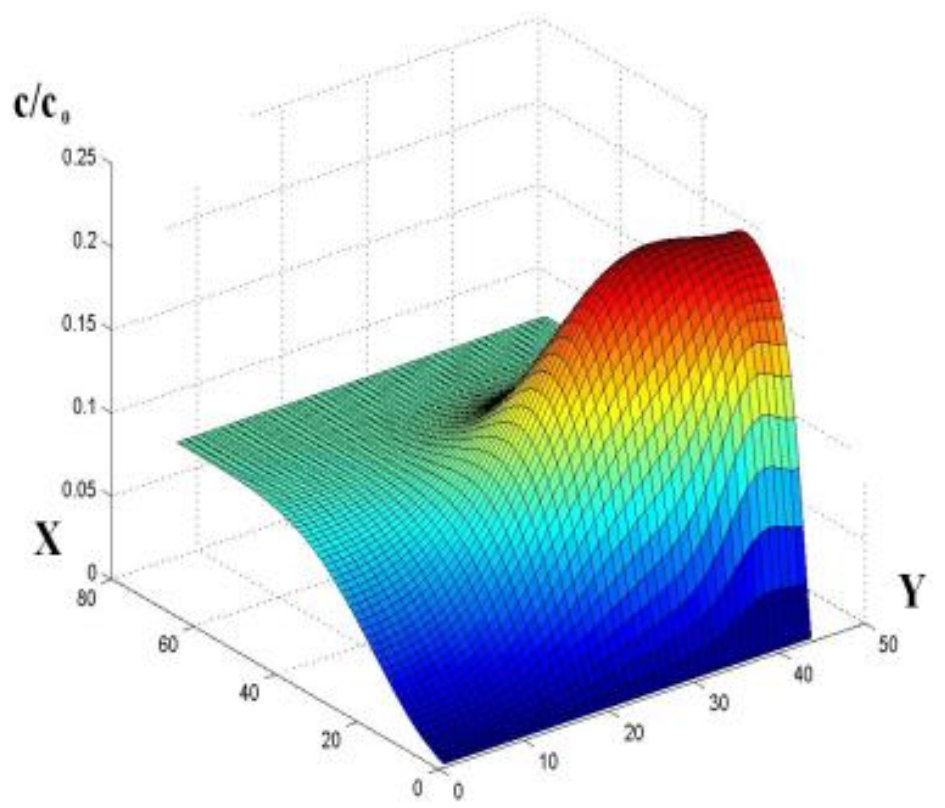

Fig. 4. (Color online) Isolines of equal concentration of $\mathrm{NaCl}$ in space for $t=35 \Delta t$. 


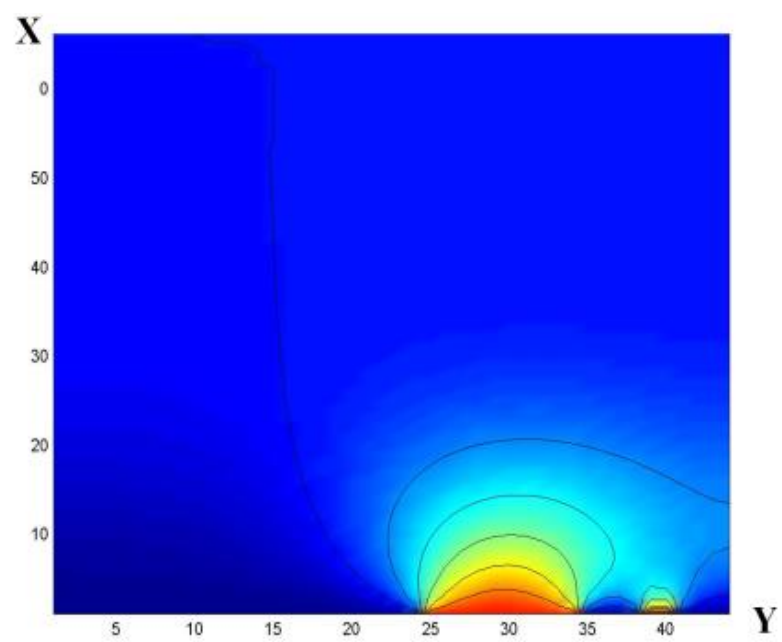

Fig. 5. (Color online) Isolines of equal concentration of $\mathrm{NaCl}$ in plan for $t=35 \Delta t$.

The following input data are used for the tests in Fig. 6:

$M_{x}=0.0457886, M_{y}=0.019554, M_{z}=0.00437, H=0.3, D_{x}=0.001157$, $D_{y}=0.029554, D_{z}=0.0052311, A_{1}=0, A_{2}=0.0076, A_{3}=1, B_{1}=0.0010$, $B_{2}=-0.0052, B_{3}=1, C_{0}=1$ for $0.55 \leq y \leq 0.66$ and $C_{0}=0.4$ for $0.91 \leq y \leq$ $0.96, t^{*}=100$.

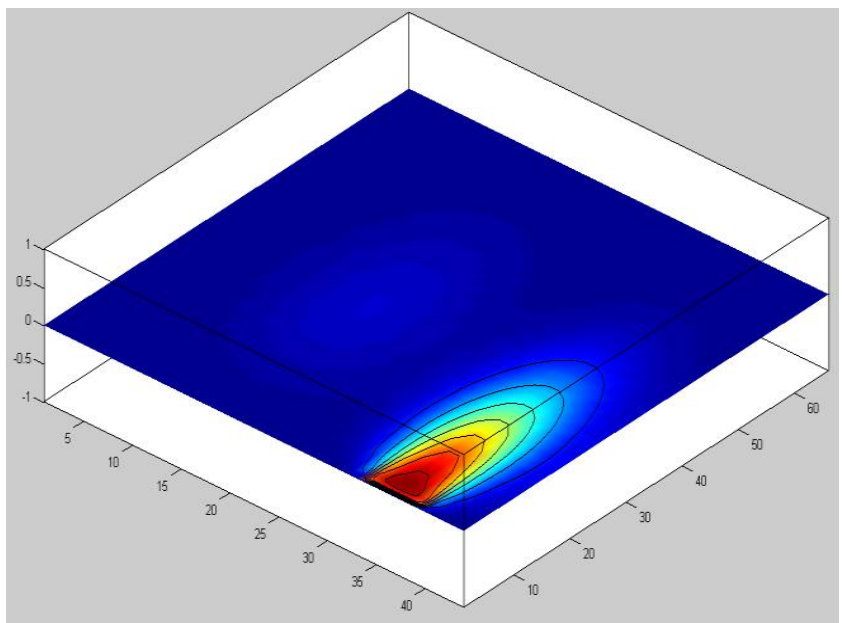

Fig. 6. (Color online) Isolines of equal concentration for pollution with ammonium nitrate in plan for $t=70 \Delta t$. 


\section{CONCLUSIONS}

The present article gives a modern concept, methodology and recommendations for practical application of the filtration thermo-dispersion of non-conservative admixtures in porous media.

An original method has been developed for numerical 3-d modeling of the problems of filtration thermo-dispersion in porous media with and without pressure-head on the basis of the final element and final difference methods.

The equations of filtration, convective dispersion, together with Darcy's law, and the equations of state are modified, improved, jointly solved and implemented in practice. An algorithm has been developed for the numerical solution of the problems, related to fertilizer $\mathrm{C}$ pollution.

On the basis of the results obtained methodological proposals are formulated for determining the effect of density and viscosity depending on the equations of state of filtration dispersion in groundwater.

The software has been developed for solving problems related to fertilizer contaminant migration in groundwater. The obtained results may be used for the classification of soils, polluted due to natural and artificial fertilizer application with the view of the quality grading of the ecological conditions. The numerical results of the software product are visualized in a Matlab environment.

\section{REFERENCES}

[1] G. Pinder, W. GRAy (1987) "Finite element simulation in surface and subsurface hydrology". Academic Press, New York.

[2] J. FRID (1981) "Groundwater Pollution". Moscow, Nedra.

[3] V. Shestakov (1979) "Groundwater Dynamics". Moscow, MGU.

[4] A. Scheidegger (1988) "The Physics of Flow through Porous Media". 3rd Edition. Univ. of Toronto Press, Toronto, Canada.

[5] R. Petkov (2008) Determining the Filtration Dispersion Coefficients in an Anisotropic Homogeneous Porous Medium. In: Conference Proceedings, Ohrid, Republic of Macedonia, pp. 237-244.

[6] A. Ravdel, A. Ponomarev (1983) "Brief Handbook of Physico-Chemical Quantities". Leningrad, Himia.

[7] R. Petkov (1990) Numerical Solution of 3-D Problems of Groundwater Pollution with a Free Boundary. In: "Contaminant Transport in Groundwater". A.A. Balkema, Rotherdam, pp. 485-492.

[8] R.M. Petkov (1992) Application of F for Solving Thermodispersion Problems in Porous Media, Theoretical and Applied Mechanics, Sofia 22(2) 71-77.

[9] M. Stohr, K. Rотн (2005) Gradient-based Estimation of Local Parameters for Flow Transport in Heterogeneous Porous Media. Resources Research 41 4110-4140.

[10] L. Willardson, R. Hurst (1965) Sample Size Estimates in Permeability Studies. Journal of the Irrigation and Drainage Division, ASCE 91 1-9. 